\title{
Optimal chemotherapy for advanced gastric cancer: is there a global consensus?
}

\author{
Florian Lordick · Sylvie Lorenzen • \\ Yasuhide Yamada $\cdot$ David Ilson
}

Received: 9 May 2013/Accepted: 17 August 2013/Published online: 19 September 2013

(C) The International Gastric Cancer Association and The Japanese Gastric Cancer Association 2013

\begin{abstract}
The optimal medical treatment for advanced gastric cancer is currently the source of debate. Cytotoxic treatment has been shown to prolong survival and provide improved symptom control compared with best supportive care alone, but a global standard has not yet been defined. A literature research was undertaken. Results were evaluated by an international author team. The conclusions of this are presented in this paper. Combination chemotherapy with cisplatin and 5-fluorouracil was the preferred first-line chemotherapy, but oxaliplatin has shown equivalent efficacy to cisplatin. Oral fluoropyrimidines, especially S-1 and capecitabine, can substitute for 5-fluorouracil. Modern doublet regimens are preferred in the majority of patients on the basis of a balanced benefit-to-risk ratio. In selected fit and compliant patients, especially those with a high tumor burden or potential secondary resectability, a third drug may be added because triplet chemotherapy led to higher responses rates and enhanced efficacy.
\end{abstract}

F. Lordick ( $\square)$

University Cancer Center Leipzig (UCCL), University Clinic Leipzig, University of Leipzig, Liebigstr. 20,

04301 Leipzig, Germany

e-mail: florian.lordick@medizin.uni-leipzig.de

S. Lorenzen

Department of Hematology and Oncology, Klinikum rechts der Isar, Technical University of Munich, Munich, Germany

Y. Yamada

Gastrointestinal Medical Oncology Division, National Cancer Center Hospital, Tokyo, Japan

D. Ilson

Department of Medicine, Gastrointestinal Oncology Unit, Memorial Sloan Kettering Cancer Center, New York, USA
However, docetaxel also adds a significant increase in side effects. Monotherapy and early dose modifications should be considered in elderly and infirm patients. Beyond that, our understanding of gastric cancer tumor biology is increasing. In HER2-positive gastric cancer, the addition of the monoclononal anti-HER2 antibody trastuzumab to cisplatin and fluoropyrimidines has prolonged survival duration. Second-line chemotherapy with single agents has now become a proven treatment option. Alternatively, anti-angiogenic treatment with ramucirumab is on the horizon. In conclusion, combination chemotherapy is regarded as the global standard of care for the first-line treatment of advanced gastric cancer. Molecularly targeted treatments are being explored, preferably in combination with a backbone of chemotherapy doublets.

Keywords Gastric cancer - Chemotherapy ·

Metastases · Consensus · Recommendation

\section{Introduction}

Gastric cancer (GC), including adenocarcinoma of the distal esophagus and the esophagogastric junction (EGJ), is a major global health problem. Around 1 million new cases and 750,000 deaths occur per year worldwide, accounting for $10 \%$ of all deaths due to cancer [1,2]. The highest incidence rates are found in East Asia, East Europe, and parts of South America, while the lowest rates occur in North America [2-4] (Fig. 1).

In Europe and North America, the overall 5-year survival for GC is approximately $25 \%$ [3], while superior outcomes with 5-year-survival rates of approximately $60 \%$ are reported in East Asia [5]. Early diagnosis due to well- 


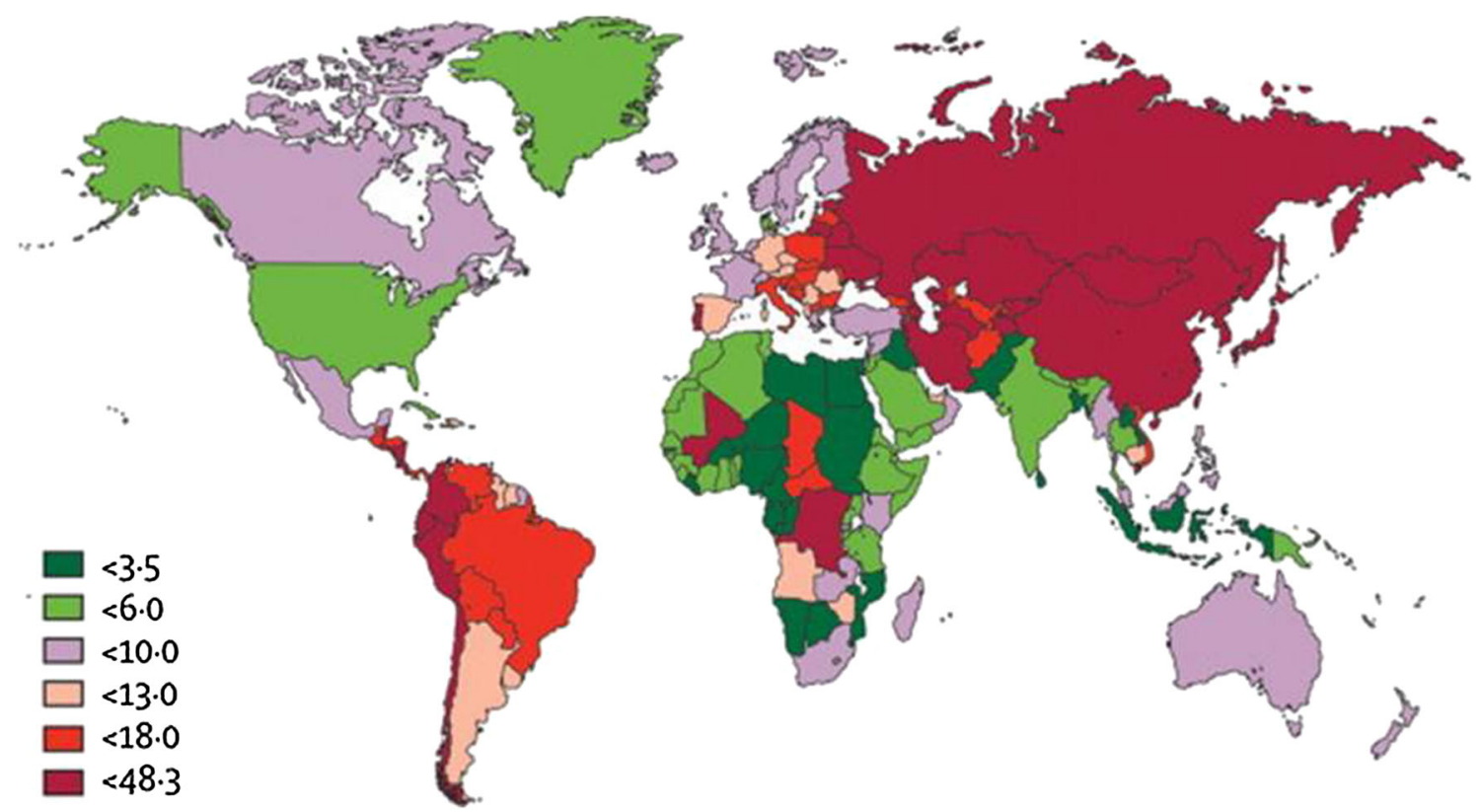

Fig. 1 Age-adjusted gastric cancer incidence per 100,000 inhabitants (according to Lozano et al. [2])

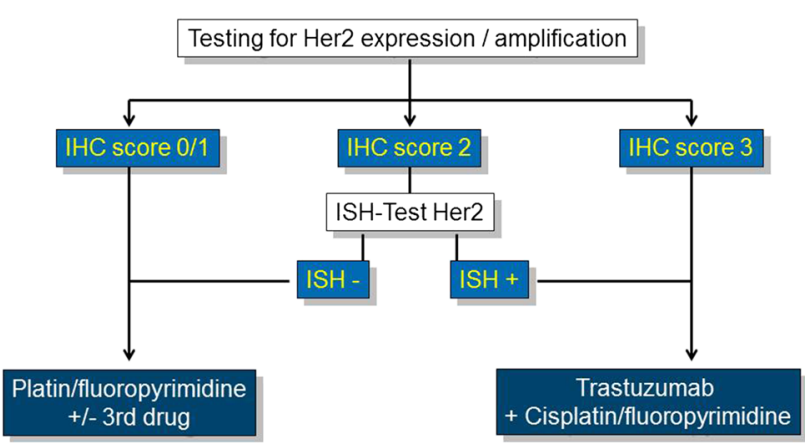

Fig. 2 HER2 testing and treatment algorithm in advanced gastric cancer. IHC immunohistochemistry, ISH in situ hybridization

established screening programs, careful surgical lymph node dissection in localized disease, and consistent use of postoperative adjuvant chemotherapy may explain some of the differences in patient outcomes [3, 6, 7]. Epidemiological studies indicate a progressive decrease in the intestinal type of gastric cancer and an increase in the diffuse type [8], while intestinal-type tumors still predominate in East Asia and East Europe. As our understanding of gastric cancer biology has improved, differential treatment approaches for specific subtypes of gastric cancer have emerged [9]. HER2-positive advanced GC is now treated by the addition of the monoclonal antibody trastuzumab to standard chemotherapy [10] (Fig. 2). More molecular characteristics are being identified and more specific and targeted treatments are being studied [11].

\section{Methods}

References for this review were identified through searches of PubMed with the search terms "chemotherapy," "gastric cancer," "esophagogastric junction cancer," "advanced," "metastatic," and "quality of life" from 1990 until April 2013. Articles were also identified through searches of the major oncology congress abstract search machines (American Society of Clinical Oncology Annual Meetings 2010-2013, European Society of Medical Oncology and European Cancer Organization Annual Meetings 2010-2012).

The benefit of chemotherapy in advanced gastric cancer

In this review, the term "advanced" indicates a disease extent that is no longer amenable to curative surgical treatment. It has been shown that chemotherapy can prolong survival in this setting [12]. This is true for first-line treatment as well as for second- and further-line chemotherapy (Table 1) [13-17]. Symptom control and quality of life have also been looked at in some studies, and have been demonstrated to be improved by chemotherapy.

For the first-line treatment of GC, it has been shown that combination chemotherapy is, in principle, more efficacious than monotherapy $[12,18,19]$. It should be noted that the benefit observed in the Cochrane review was rather marginal [hazard ratio (HR) for survival of $0.82 ; 95 \% \mathrm{CI}$ $0.74-0.90]$. In addition, toxicity increases with combination schedules. Therefore, careful evaluation of the patient's performance status and the different toxicity 
Table 1 Phase III trials of chemotherapy versus best supportive care (BSC) for advanced gastric cancer

\begin{tabular}{|c|c|c|c|c|c|c|}
\hline Study & Setting & $\begin{array}{l}\text { Number of } \\
\text { patients }\end{array}$ & Treatment & $\begin{array}{l}\text { Response } \\
\text { rate }(\%)\end{array}$ & $\begin{array}{l}\text { Median overall } \\
\text { survival (months) }\end{array}$ & $\begin{array}{l}\text { Quality } \\
\text { of life }\end{array}$ \\
\hline Pyrhönen et al. [13] & 1st line & $\begin{array}{l}21 \\
20\end{array}$ & FEMTX vs. BSC & 29 & 12.3 vs. $3.1(P=0.0006)$ & - \\
\hline Murad et al. [14] & 1st line & $\begin{array}{l}30 \\
10\end{array}$ & FAMTX vs. BSC & 50 & 9 vs. $3(P=0.001)$ & - \\
\hline Glimelius et al. [15] & 1st line & $\begin{array}{l}31 \\
30\end{array}$ & ELF vs. BSC & NR & 8 vs. 5 (NS) & $\begin{array}{l}\text { In favor } \\
\text { of ELF }\end{array}$ \\
\hline Thuss-Patience et al. [16] & 2nd line & 40 & Irinotecan vs. BSC & $\begin{array}{c}0 \text { (58 stable } \\
\text { disease) }\end{array}$ & 4 vs. $2.4(P=0.0023)$ & - \\
\hline Kang et al. [17] & 2nd line & 202 & $\begin{array}{l}\text { Irinotecan or docetaxel vs. } \\
\text { BSC }\end{array}$ & 6 & 5.3 vs. $3.8(P=0.007)$ & - \\
\hline
\end{tabular}

FEMTX fluorouracil/epidoxorubicin/methotrexate, FAMTX fluorouracil/doxorubicin/methotrexate, ELF etoposide/leucovorin/fluorouracil, NR not reported, $N S$ not significant

profiles of the treatment regimens should be performed before choosing the therapy. Although the majority of patients are $\geq 65$ years old, elderly patients are generally underrepresented in clinical trials, mainly due to concerns regarding toxicity. Moreover, elderly patients who are enrolled in clinical studies may not represent the typical characteristics of an elderly GC population. Analyzing data from three randomized controlled trials, there were no significant differences in the incidence of grade 3/4 toxicity between younger and elderly adults. In terms of response rates, failure-free and overall survival elderly patients did benefit from chemotherapy to a similar degree as younger patients. In a multivariate analysis, independent prognostic factors for survival were performance status and locally advanced disease, but not age [20]. Nevertheless, careful assessment of functional status and comorbidities before the start of therapy is highly recommended, and the selection of sequential one-, two-, or three-drug regimens should be evaluated individually.

Systemic chemotherapy can prolong survival, improve symptom control and stability, and potentially improve quality of life. Combinations are more effective than single-agent chemotherapy and can also be recommended for elderly patients after proper evaluation of performance status and comorbidities. A combination comprising a platinum compound and a fluoropyrimidine can be regarded as an accepted first-line practice.

Which platinum compound should be used?

Cisplatin has been an integral part of GC reference regimens globally [12]. Due to its specific side effects, including nephrotoxicity, ototoxicity, and emetogenicity, other platinum salts have been studied. Carboplatin did not exhibit sufficient activity in phase II studies and was therefore not studied any further in randomized controlled trials [21]. In contrast, oxaliplatin, which had improved the efficacy of 5-fluorouracil (5-FU) treatment in colorectal cancer, was extensively studied in GC. Following promising phase II study results [22-24], oxaliplatin was compared with cisplatin in two randomized controlled trials. Both studies were designed to prove the non-inferiority of oxaliplatin compared with cisplatin. The Randomized ECF for Advanced and Locally Advanced Esophagogastric Cancer2 (REAL-2) study had a two-by-two design. One thousand two patients were included, who received epirubicin/cisplatin plus either 5-FU (ECF) or capecitabine (ECX) or epirubicin/oxaliplatin plus either 5-FU (EOF) or capecitabine (EOX). For the oxaliplatin-cisplatin comparison, the hazard ratio for the oxaliplatin group [0.92 (95\% confidence interval CI, 0.80-1.10)] proved that oxaliplatin is non-inferior to cisplatin. As compared with cisplatin, oxaliplatin was associated with lower incidences of neutropenia, alopecia, renal toxicity, and thromboembolism, but with slightly higher incidences of diarrhea and neuropathy [25]. At the same time, the German Arbeitsgemeinschaft Internistische Onkologie (AIO) compared 5-FU/leucovorin and cisplatin (FLP) with 5-FU/leucovorin and oxaliplatin (FLO) [26]. The AIO study found a trend towards improved progression-free survival (PFS) with FLO versus FLP in 220 randomized patients, but no significant difference in overall survival (OS). Remarkably, FLO was associated with significantly less toxicity, including anemia, nausea, vomiting, alopecia, fatigue, renal toxicity, thromboembolic events, and other serious treatment-related adverse events. Sensory neuropathy was more common in the oxaliplatin group. In patients aged $>65$ years $(n=92)$, treatment with oxaliplatin resulted in significantly superior PFS (6.0 vs. 3.1 month; $P=0.029)$ and improved OS (13.9 vs. 7.2 months) as compared with cisplatin.

Cisplatin plus S-1 (CS) is the standard first-line treatment regimen for advanced gastric cancer in Japan [19]. 
Oxaliplatin plus S-1 (SOX) showed non-inferiority to CS in PFS [27]. The median PFS was 5.5 months for SOX vs. 5.4 months for CS (hazard ratio 1.00; $95 \%$ CI, 0.84-1.20). The response rate was $56 \%$ for SOX and $52 \%$ for CS $\left(\chi^{2}\right.$ test, $P=0.37$ ). The most common grade $3 / 4$ toxicities in SOX vs. CS were neutropenia 19.5 vs. $41.5 \%$, thrombocytopenia 9.5 vs. $10.4 \%$, febrile neutropenia 0.9 vs. $6.9 \%$, and anorexia 14.8 vs. $18.5 \%$, respectively. Accordingly, SOX is considered a new standard option for first-line treatment in Japan.

In conclusion, oxaliplatin is generally less toxic than cisplatin. In view of its non-inferior efficacy, oxaliplatin can substitute for cisplatin in the treatment of advanced GC. Elderly patients may derive a particular benefit from treatment with oxaliplatin instead of cisplatin. However, oxaliplatin has not been approved in Europe, North America, or Japan by the medicine agencies and is therefore not reimbursed for the treatment of $\mathrm{GC}$ in some countries. In Korea, capecitabine-oxaliplatin or 5-FU, leucovorin, plus oxaliplatin is reimbursed and frequently used as first-line treatment for advanced GC.

Can oral fluoropyrimidines substitute for 5-FU?

Intravenous 5-FU has been the standard combination partner for platinum salts and other cytotoxic compounds in the treatment of GC.

The REAL-2 study compared capecitabine, an orally available 5-FU prodrug, with intravenous 5-FU [25]. The fluoropyrimidine comparison in REAL-2 showed noninferiority of capecitabine with a hazard ratio for death of 0.86 (95\% CI, 0.80-0.99). The ML17032 study was performed in parallel in Korea and included 316 patients who were randomly assigned to receive either cisplatin/5-FU or cisplatin/capecitabine. The response rate was significantly higher in the capecitabine group (42 vs. $32 \%, p=0.02$ ). The survival analysis proved the non-inferiority of capecitabine [28]. In a combined analysis of REAL-2 and ML17032, OS was even superior in patients treated with capecitabine combinations compared with patients treated with 5-FU; HR 0.87 (95 \% CI 0.77-0.98, $P=0.02$ ) [29]. However, the reported substantial toxicity of hand foot syndrome (HFS), which $22 \%$ experienced with capecitabine compared to only $4 \%$ with 5FU in the ML17032 study [28], and $46 \%$ compared to $29 \%$ all-grade HFS with capecitabine compared to 5FU in the REAL-2 study [25], may undercut the potential advantage of oral over continuous infusion administration.

Another oral fluoropyrimidine, S-1, is now approved in East Asia and Europe for the treatment of advanced GC. S-1 contains tegafur/gimeracil/oteracil potassium in a molar ratio of 1.0:0.4:1.0. Gimeracil reduces the degradation of 5-FU and oteracil improves its gastrointestinal tolerability. S-1 in combination with cisplatin has been established as the standard first-line chemotherapy in advanced GC in Japan (SPIRITS trial) [19]. S-1 was also shown to be non-inferior to infusional 5-FU when both were given as single agents [30]. With a dose established in a Western patient population [31] and with the hypothesis that cisplatin/S-1 could improve overall survival, safety, and convenience compared to cisplatin/5-FU, a randomized comparison was attempted in a non-Asian phase III trial into which 1053 patients were enrolled. The median OS was 8.6 months in the cisplatin/S-1 arm and 7.9 months in the cisplatin/5-FU arm, showing no significant difference. However, significant safety advantages were observed with S-1/cisplatin for the rates of complicated neutropenia, stomatitis, hypokalemia, and treatment-related deaths [32]. Note that cisplatin was administered at a reduced dosage in the $\mathrm{S}-1$ arm $\left(75 \mathrm{mg} / \mathrm{m}^{2}\right)$ compared to the standard arm $\left(100 \mathrm{mg} / \mathrm{m}^{2}\right)$, possibly explaining the more favorable toxicity profile with $\mathrm{S} 1 /$ cisplatin. The results of a randomized trial proving the efficacy of S-1 and oxaliplatin in Japanese patients treated for advanced gastric cancer has already been reported [33]. Nevertheless, due to considerable pharmacokinetic differences when used in non-Asians, clinical experience with S-1 in Western countries suggests a different toxicity than that reported in Asian populations. Several polymorphisms have been identified in genes encoding drug-metabolizing enzymes, which may explain this differential toxicity of fluoropyrimidines between Asian and Western populations [34].

Oral fluoropyrimidines can substitute for intravenous 5-FU and are now subsidized for advanced GC in most countries. Although no superior survival was shown with the combination cisplatin/S-1, significant safety advantages were observed compared to cisplatin/5-FU. Cisplatin and capecitabine has become the standard backbone chemotherapy in trials investigating monoclonal antibodies in GC $[10,35,36]$. In the treatment of elderly or frail patients, or in cases where platinum agents are contraindicated, singleagent fluoropyrimidine, although not as effective as doublet regimens, should also be considered an option [37, 38].

Doublets or triplets-the rationale for adding a third cytotoxic drug

Triplet combination chemotherapy comprising an anthracycline or a taxane in addition to a platinum compound and a fluoropyrimidine has resulted in higher response rates and a modest improvement in overall survival compared with doublet combinations, but it also exposes patients to more serious side effects [12, 19]. A variety of treatment regimens have been established in randomized phase III studies which are the standard of care in different parts of the world, but not globally (Table 2). 
Table 2 First-line treatment regimens developed in randomized controlled trials in advanced gastric cancer

\begin{tabular}{|c|c|c|c|c|c|c|}
\hline $\begin{array}{l}\text { Chemotherapy } \\
\text { agents }\end{array}$ & $\begin{array}{l}\text { Dosage } \\
\left(\mathrm{mg} / \mathrm{m}^{2}\right)\end{array}$ & Application & Setting & $\begin{array}{l}\text { Response } \\
\text { rate }(\%)\end{array}$ & $\begin{array}{l}\text { Median PFS } \\
\text { (months) }\end{array}$ & $\begin{array}{l}\text { Median OS } \\
\text { (months) }\end{array}$ \\
\hline \multicolumn{7}{|l|}{ Triplet combinations } \\
\hline ECF [25] & & & 1st line & 40.7 & 6.2 & 9.9 \\
\hline Epirubicin & 50 & i.v. day 1 & & & & \\
\hline Cisplatin & 60 & i.v. day 1 & & & & \\
\hline 5-Fluorouracil & 200 & i.v. continuous infusion day $1-21$ & & & & \\
\hline \multicolumn{7}{|l|}{ Q3w } \\
\hline ECX [25] & & & 1st line & 46.4 & 6.7 & 9.9 \\
\hline Epirubicin & 50 & i.v. day 1 & & & & \\
\hline Cisplatin & 60 & i.v. day 1 & & & & \\
\hline Capecitabine & 1250 & p.o. day $1-21$ & & & & \\
\hline \multicolumn{7}{|l|}{ Q3w } \\
\hline EOF [25] & & & 1st line & 42.4 & 6.5 & 9.3 \\
\hline Epirubicin & 50 & i.v. day 1 & & & & \\
\hline Oxaliplatin & 130 & i.v. day 1 & & & & \\
\hline 5-Fluorouracil & 200 & i.v. continuous infusion day $1-21$ & & & & \\
\hline \multicolumn{7}{|l|}{ Q3w } \\
\hline EOX [25] & & & 1st line & 47.9 & 7.0 & 11.2 \\
\hline Epirubicin & 50 & i.v. day 1 & & & & \\
\hline Oxaliplatin & 130 & i.v. day 1 & & & & \\
\hline Capecitabine & 1250 & p.o. day 1-21 & & & & \\
\hline \multicolumn{7}{|l|}{ Q3w } \\
\hline $\mathrm{DCF}[41]$ & & & 1st line & 37.0 & 5.6 & 9.2 \\
\hline Docetaxel & 75 & i.v. day 1 & & & & \\
\hline Cisplatin & 75 & i.v. day 1 & & & & \\
\hline 5-Fluorouracil & 750 & i.v. day $1-5$ & & & & \\
\hline \multicolumn{7}{|l|}{ Q3w } \\
\hline \multicolumn{7}{|l|}{ Doublet combinations } \\
\hline FLO [26] & & & 1st line & 34.8 & 5.8 & 10.7 \\
\hline Oxaliplatin & 85 & i.v. day 1 & & & & \\
\hline Folinic acid & 200 & i.v. day 1 & & & & \\
\hline 5-Fluorouracil & 2600 & i.v. $24 \mathrm{~h}$ & & & & \\
\hline \multicolumn{7}{|l|}{ Q2w } \\
\hline FLP [26] & & & 1st line & 24.5 & 3.9 & 8.8 \\
\hline Cisplatin & 50 & i.v. day 1 & & & & \\
\hline Folinic acid & 200 & i.v. day 1 & & & & \\
\hline 5-Fluorouracil & 2000 & i.v. $24 \mathrm{~h}$ & & & & \\
\hline \multicolumn{7}{|l|}{ Q2w } \\
\hline Cisplatin/capecitabine [28] & & & 1st line & 46.0 & 5.6 & 10.5 \\
\hline Cisplatin & 80 & i.v. day 1 & & & & \\
\hline Capecitabine & 2000 & p.o. day $1-14$ & & & & \\
\hline \multicolumn{7}{|l|}{ Q3w } \\
\hline Western cisplatin $+\mathrm{S} 1$ [32] & & & 1stline & 29.1 & 4.8 & 8.6 \\
\hline Cisplatin & 75 & i.v. day 1 & & & & \\
\hline S1 & 50 & p.o. day $1-21$ & & & & \\
\hline \multicolumn{7}{|l|}{ Q4w } \\
\hline Asian cisplatin + S1 [19] & & & 1st line & 54 & 6.0 & 13.0 \\
\hline Cisplatin & 60 & i.v. day 8 & & & & \\
\hline S1 & $40-60 \mathrm{mg}$ & p.o. day $1-21$ & & & & \\
\hline
\end{tabular}


Table 2 continued

\begin{tabular}{|c|c|c|c|c|c|c|}
\hline $\begin{array}{l}\text { Chemotherapy } \\
\text { agents }\end{array}$ & $\begin{array}{l}\text { Dosage } \\
\left(\mathrm{mg} / \mathrm{m}^{2}\right)\end{array}$ & Application & Setting & $\begin{array}{l}\text { Response } \\
\text { rate }(\%)\end{array}$ & $\begin{array}{l}\text { Median PFS } \\
\text { (months) }\end{array}$ & $\begin{array}{l}\text { Median OS } \\
\text { (months) }\end{array}$ \\
\hline \multicolumn{7}{|l|}{ Q5w } \\
\hline Asian oxaliplatin + S1 [27] & & & 1st line & 56 & 5.5 & Not available \\
\hline Oxaliplatin & $100 \mathrm{mg} / \mathrm{m}^{2}$ & i.v. day 1 & & & & \\
\hline S1 & $40-60 \mathrm{mg}$ & p.o. day $1-14$ & & & & \\
\hline Q3w & & & & & & \\
\hline
\end{tabular}

i.v. intravenous, $O S$ overall survival, $P F S$ progression-free survival, p.o. per os

\section{Anthracycline-containing triplet combinations}

The literature is inconsistent regarding the potential value of anthracyclines. In the UK and in some other countries, the incorporation of anthracyclines into the palliative medical treatment of gastric cancer has become common practice. According to the Cochrane analysis, randomized studies have proven the value of anthracyclines given in addition to platinum and 5-FU [12]. However, the evidence to support the activity of an anthracycline-based triplet [i.e., epirubicin, cisplatin, and 5-fluorouracil (ECF)] is provided by only three randomized studies, with a total sample size of 501 patients [12]. Note that the largest study included in this meta-analysis is a comparison between ECF and another triplet (mitomycin C plus CF, MCF) [39], which may lead us to question the conclusion that the addition of an anthracycline improves outcome, presuming that mitomycin $\mathrm{C}$ had a negative effect on $\mathrm{CF}$ efficacy. More recently, a relatively small randomized trial from Korea could not demonstrate improved efficacy upon the addition of epirubicine to cisplatin and capecitabine (ECX) compared to only cisplatin and capecitabine (CX) [40]. Despite the uncertainty regarding the value of adding an anthracycline to cisplatin and 5-FU, this anthracycline triplet remains the standard treatment in the UK. The combination of epirubicine, oxaliplatin, and capecitabine (EOX regimen) has shown superior survival compared with ECF in the randomized REAL-2 study (ECF) [25] (9.9 vs. 11.2 months; HR: $0.8 ; p=0.02$ ); however, a comparison to common two-drug regimens such as $\mathrm{CF}$ is lacking.

\section{Docetaxel-containing triplet combinations}

The randomized controlled TAX 325 trial showed a significantly improved overall survival (median 9.2 vs. 8.6 months; HR 1.29; $P=0.0021$ ) for the addition of docetaxel (DCF) compared with cisplatin/5-FU (CF). Secondary endpoints were response rate (37 vs. $25 \%)$ and time to progression (5.6 vs. 3.7 months; HR1.47; $P=0.0004$ ), which were also in favor of DCF [41]. The median age of the patients included was 55 years, with only $24 \%$ of patients aged $\geq 65$. The majority of the patients had a good Karnofsky performance status ( $\geq 90: 64 \%$ ). However, DCF was associated with substantial toxicity, particularly myelosuppression, with a $29 \%$ incidence of febrile neutropenia, and gastrointestinal side effects were markedly increased (49\% grade $3 / 4$ ). As a consequence, half of the patients discontinued treatment with DCF for either adverse events or patient refusal. Given that patients in clinical trials are usually carefully selected to be of a younger age and to have near-optimal organ and functional status, and thus do not necessarily reflect the geriatic and frail patients more commonly treated in clinical practice, the routine use of this toxic DCF regimen is rather questionable [41]. Due to the high rates of hematologic and other toxicities observed with the original DCF regimen, alternative docetaxel-containing regimens have been investigated in several phase II studies (Table 3). The principle of splitting docetaxel from 3-weekly into weekly or bi-weekly administration has led to a considerable decrease in hematological toxicity. Although evidence from phase III studies is lacking, the modification of DCF by alternative scheduling has decreased the toxicity, apparently without compromising the efficacy. Therefore, if docetaxel-based first-line treatment is considered, one should refrain from using classic DCF and choose an alternative treatment protocol such as Gastro-Tax [42], FLOT [43], or ATTAX [44]. Regarding the use of an intensive docetaxel-based triplet combination in elderly patients ( $\geq 65$ years), the FLOT combination appears to be feasible and effective but no better than FOLFOX, although toxicity was markedly increased and quality of life was negatively impacted in a significant proportion of patients during the first 8 weeks of treatment [45]. Therefore, despite all of the associated improvements, docetaxel-containing treatment regimens should be only considered in fit and compliant patients, and proper patient selection-including critical evaluation of performance status and comorbidities, as well as access to frequent assessment of toxicity-should be performed before the onset of therapy. An alternative highly active and tolerable doublet chemotherapy regimen is the combination of docetaxel and $\mathrm{S}-1$, which showed a promising median overall survival of 14.3 months and a median TTP of 
Table 3 Docetaxel-containing combination regimens: phase II/III

\begin{tabular}{|c|c|c|c|c|c|c|}
\hline Study & Phase & $\begin{array}{l}\text { Number of } \\
\text { patients }\end{array}$ & Regimen & $\begin{array}{l}\text { Overall response } \\
\text { rate }(\%)\end{array}$ & $\begin{array}{l}\text { Median PFS } \\
\text { (months) }\end{array}$ & $\begin{array}{l}\text { Median OS } \\
\text { (months) }\end{array}$ \\
\hline van Cutsem et al. [41] & III & 224 & DCF & 37 & 5.6 & 9.2 \\
\hline Q3w & & 221 & $\mathrm{CF}$ & 25 & 3.7 & 8.6 \\
\hline Roth et al. [54] & II & 61 & TCF & 41 & 4.6 & 10.4 \\
\hline \multirow[t]{2}{*}{ Q3w } & & 59 & $\mathrm{TC}$ & 38 & 3.6 & 11.0 \\
\hline & & 58 & ECF & 40 & 4.9 & 8.3 \\
\hline Tebbutt et al. [44] & II & 50 & wDCF & 47 & 5.9 & 11.2 \\
\hline Q3w & & 56 & wDX & 26 & 4.6 & 10.1 \\
\hline Shah et al. [69] & II & 30 & $\mathrm{mDCF}$ & 52 & NR & 15.1 \\
\hline Q3w & & 31 & $\mathrm{DCF}+\mathrm{G}-\mathrm{CSF}$ & 34 & NR & 12.6 \\
\hline Van Cutsem et al. [70] & II & 79 & TE Q3w & 23.1 & 4.5 & 9.0 \\
\hline \multirow[t]{2}{*}{ Q2w/Q3w } & & 89 & TEF Q2w & 46.6 & 7.7 & 14.6 \\
\hline & & 86 & TEX Q3w & 25.6 & 5.6 & 11.3 \\
\hline Al-Batran et al. [43] & II & 54 & FLOT & 58 & 5.2 & 11.1 \\
\hline \multicolumn{7}{|l|}{ Q2w } \\
\hline Lorenzen et al. [42] & II & 60 & T-PLF & 47 & 8.1 & 15.1 \\
\hline \multicolumn{7}{|l|}{ Q2w } \\
\hline Yoshida et al. [46] & II & 48 & DS & 56.3 & 7.3 & 14.3 \\
\hline \multicolumn{7}{|l|}{ Q3w } \\
\hline Koizumi et al. [48] & II & 59 & DCS & 81 & 8.7 & 18.5 \\
\hline \multicolumn{7}{|l|}{ Q4w } \\
\hline Yoshida et al. [47] & III & 314 & DS & 38.8 & 5.3 & 12.5 \\
\hline Q3w & & 314 & $\mathrm{~S}$ & 26.8 & 4.2 & 10.8 \\
\hline
\end{tabular}

$P F S$ progression-free survival, $O S$ overall survival, $E C F$ epirubicin/cisplatin/FU, $E C X$ epirubicin/cisplatin/capecitabine, $E O F$ epirubicin/oxaliplatin/FU, EOX epirubicin/oxaliplatin/capecitabine, TE docetaxel/oxaliplatin, $T E F$ docetaxel/oxaliplatin/FU, TEX docetaxel/oxaliplatin/capecitabine, FLOT docetaxel/oxaliplatin/FU/leucovorin, T-PLF docetaxel/cisplatin/FU/leucovorin, $D C F$ docetaxel/cisplatin/FU, $D X$ docetaxel/ capecitabine, $D F$ docetaxel/FU, $m$ modified, $D C S$ docetaxel/cisplatin/S-1, $D S$ docetaxel/S-1, G-CSF granulocyte colony-stimulating factor, $w$ weekly

8.3 months in a single center in Asia [46]. This trial was the basis for the randomized phase III START trial comparing docetaxel/S-1 with $\mathrm{S}-1$ in patients with advanced gastric cancer. An updated analysis presented at ESMO 2012 showed an improved median survival time of 12.5 months in the combination therapy group compared to 10.8 months in patients who received $\mathrm{S}-1$ alone $(p=0.0319)$ [47]. Another Japanese approach involving a triplet regimen was evaluated in a phase I/II trial in which patients received $S-1$, docetaxel $\left(40 \mathrm{mg} / \mathrm{m}^{2}\right.$ on day 1$)$, and cisplatin $\left(60 \mathrm{mg} / \mathrm{m}^{2}\right.$ on day 1$)$ (DCS), or S-1 $(80-120 \mathrm{mg} /$ day), 2 weeks on, 2 weeks off, every 4 weeks [48]. The most commonly observed grade $3 / 4$ toxicity was neutropenia in $70 \%$ of patients. The gastrointestinal toxicities were very low. The median PFS was 8.7 months and the median survival was 18.5 months. DCS is also being compared with CS in an ongoing phase III trial in Japan (JCOG 1013), from which known HER2-positive patients are excluded. The study aims to recruit a total of 740 patients and the primary endpoint is overall survival. The patients will be stratified according to institution, number of metastatic sites, measurable or nonmeasurable, and diffuse or intestinal type. The key secondary endpoint is survival by histology.

Irinotecan-containing regimens

Irinotecan-based combination regimens have been studied as a first-line alternative to platinum-based chemotherapy. The first phase II study results suggested that irinotecan and 5-fluorouracil combinations had promising antitumoral activity and efficacy $[49,50]$. A randomized controlled phase III trial failed to show the superiority of irinotecan and high-dose 5-fluorouracil over only cisplatin and 5-fluorouracil [51]. However, quality of life tended to be better during treatment with irinotecan and 5-fluorouracil. Due to the lack of superior efficacy, irinotecan was not approved for the first-line treatment of gastric cancer in many health systems, but can be used as a "reserve regimen" with proven efficacy. Capecitabine could substitute for 5-fluorouracil as a combination partner of irinotecan [52]. 
Recommended regimens

Both doublet and triplet drug-regimens based on a platinum compound and a fluoropyrimidine can be used for the medical treatment of advanced GC. However, careful consideration of the potential toxic complications, impairment of the patient's quality of life, and the relative benefit should be undertaken. An indication for using three drugs in the first-line treatment is the presence of severe tumor symptoms, life-threatening tumor manifestations leading to the need for an instant tumor response, and the patient's preference for receiving the most active drug combination (and acceptance of enhanced side effects). Preferred regimens are the anthracycline-containing EOX regimen and the different modifications of DCF.

If doublet chemotherapy is chosen, one should be aware of the considerable toxicity associated with older high-dose cisplatin-based regimens. The $\mathrm{CF}$ regimen used in the control arm of the TAX 325 trial and other trials, cisplatin $100 \mathrm{mg} / \mathrm{m}^{2}$ and a 5-day infusion of $1000 \mathrm{mg} / \mathrm{m}^{2} 5-\mathrm{FU}$ every 4 weeks, was associated with substantial grade $3 / 4$ toxicity, mainly neutropenia (57\%), stomatitis $(27 \%)$, diarrhea $(8 \%)$, nausea $(17 \%)$, and vomiting $(17 \%)$. Newer modified regimens using a weekly or biweekly infusion schedule of 5-FU combined with either biweekly cisplatin $\left(50 \mathrm{mg} / \mathrm{m}^{2}\right)$ or oxaliplatin $\left(85 \mathrm{mg} / \mathrm{m}^{2}\right)$ demonstrated markedly reduced toxicity [26] (Table 2), indicating that these regimens should be preferred in the treatment of advanced GC. Cisplatin/capecitabine (XP), cisplatin/S-1 or oxaliplatin plus a fluoropyrimidine (FLO, CapOx, or SOX) also represent more tolerable alternatives (Table 2), with dose reductions and various supportive measures considered in the case of severe toxicity.

Alternatively, although not as effective as combination therapy, single-agent fluoropyrimidines show activity in $\mathrm{GC}$, and thus first-line fluoropyrimidine monotherapy (oral or infusional) should be considered as a reasonable option in the treatment of elderly patients or patients in whom platinum agents are contraindicated.

\section{Quality of life}

There are few reliable data on the quality of life associated with cytotoxic treatment of advanced GC. An analysis from the TAX-325 study shows that—despite being associated with considerable toxicity-DCF led to a prolongation of the time until definitive deterioration of the "global health status" as assessed by the European Organization of Research and Treatment of cancer (EORTC) quality of life C30 questionnaire [53]. This indicates that, in advanced GC, the global health status is very much influenced by the burden of disease.
Comparing docetaxel-based triplet chemotherapy with anthracycline-based therapy indicated a higher treatment burden and a worse health status/QOL for docetaxel compared to anthracycline-based therapy [54]. Several studies with ECF confirmed that improved global QOL scores were obtained compared to DCF or MCF therapy in the first 6 months of treatment $[39,54]$. Nevertheless, high treatment intensity over longer periods of time in patients with GC may again worsen health status and quality of life. Therefore, the clinical concept of starting with intensive induction regimens that reduce the disease burden followed by less intensive and better tolerated maintenance regimens that prolong the time to symptomatic tumor progression should be explored.

\section{Biologically targeted therapy}

Only modest progress has been made with novel chemotherapy agents such as oxaliplatin, docetaxel, capecitabine, and S1. Therefore, in order to further improve outcome, the identification of certain pathways that are key to cancer development is of the utmost importance. A number of biological therapies aim to inhibit components of signal transduction pathways that are amplified or functionally activated by specific genetic or epigenetic alterations. Pathways with targeted therapies where data are available or which are currently under clinical evaluation comprise HER2, VEGF, EGFR, mTOR, and c-Met.

The human epidermal growth factor receptor 2 (HER2) is overexpressed in approximately $20 \%$ of GC patients. In HER2-positive advanced GC, the international phase III Trastuzumab for Gastric Cancer (ToGA) study showed a significant improvement in the median OS of patients upon the addition of trastuzumab to cisplatin and fluoropyrimidine backbone therapy [10]. Trastuzumab in combination with chemotherapy is now a new reference treatment for the first-line treatment of HER2-positive GC. Note that the appropriate selection of patients for anti-HER2 treatment is highly dependent on the quality of HER 2 assessment by immunohistochemistry (IHC) and on the evaluation of HER2 gene amplification by in situ hybridization (ISH) techniques. Testing for HER2 in GC has its pitfalls and challenges. Optimal tumor samples should be used, and testing should be done in well-trained and quality-assured pathology laboratories [55, 56]. The greatest benefitof using trastuzumab may be gained by patients with the highest degree of HER2 overexpression: those that are IHC $3+$ or IHC $2+$ and FISH+.

Lapatinib, an oral tyrosine kinase inhibitor against both EGFR and HER2, has modest single activity in the firstline setting [57]. Results of the randomized phase III TRIO-013/Logic trial were recently presented [58]. The Logic trial could not demonstrate a statistically significant 
improvement in overall survival (primary endpoint) with the addition of lapatinib to capecitabine plus oxaliplatin (CapeOx) as the first-line treatment of advanced or metastatic HER2 + gastric or gastroesophageal adenocarcinoma (12.2 vs. 10.5 months; HR: 0.91 (95\% CI 0.73, 1.12, $p=0.35)$ ). However, pre-specified subgroup analyses showed significant improvements in OS in Asian patients $(\mathrm{HR}=0.68)$ and those under 60 years $(\mathrm{HR}=0.69)$. With regard to toxicity, lapatinib in combination with CapeOx showed an increased rate of grade 3 diarrhea (12 vs. $3 \%$ ) and a higher rate of skin toxicity. The next steps in HER2 blockade for GC may follow the developments in breast cancer, with evaluation of TDM-1, a conjugate molecule combining trastuzumab with an antimicrotubule agent; the combination of trastuzumab and lapatinib; and first-line integration of pertuzumab in metastatic disease and the investigational use of HER2 inhibitors in the neoadjuvant setting. RTOG trial 1010 is currently evaluating preoperative chemoradiotherapy in esophageal and GEJ cancers that are HER2+, randomizing patients to receive chemoradiotherapy alone or chemoradiotherapy plus trastuzumab followed by adjuvant trastuzumab after surgery.

In contrast to the success obtained with trastuzumab in advanced GC, monoclonal antibodies that target HER1 (epidermal growth factor receptor, EGFR) have failed to improve outcome in biologically unselected GC patients $[36,59]$. It remains to be elucidated from tumor tissue analyses if a small proportion of GC patients may benefit from anti-EGFR targeted therapy, e.g., in the case of EGFR gene amplification [60]. The negative results obtained with cetuximab (EXPAND study) and panitumumab (REAL3 study) emphasize the need to have a biologically meaningful target before studying targeted agents in larger populations. The importance of combining targeted agents with an appropriate chemotherapy backbone is also highlighted. As the REAL3 study taught us, the combination of a triplet chemotherapy (EOX) regimen with panitumumab is suboptimal and associated with inferior survival, probably due to the excessive toxicity experienced when using this chemotherapy backbone.

Other potential targets, including hepatocyte growth factor receptor (c-Met), insulin-like growth factor receptor 1 (IGF-1R), fibroblast growth factor receptor (FGFR), proteins involved in cell cycle regulation, the proteasome, chaperone proteins, matrix metalloproteinases, histone deacetylases, and other structures, are under evaluation. Novel drugs directed against those specific targets are under clinical investigation.

With regards to angiogenesis, the phase III AVAGAST trial [35] could not demonstrate a survival benefit with the addition of bevacizumab, an inhibitor of the ligand for vascular endothelial growth factor receptor 2 (VEGFr2), VEGF-A, to chemotherapy, but it did show improved PFS and RR. However, looking at the American and European patients, there appears to be a modest benefit of using bevacizumab, which highlights how the biology of gastric cancer varies in different parts of the world. A subsequent report suggested that high serum levels of VEGF-A and low tumor neuropilin expression were correlated with the enhanced benefit resulting from treatment with bevacizumab, but only in Western patients treated in the trial [61]. Recently, ramucirumab, a monoclonal antibody directed against VEGFR2 [62], has been shown to prolong survival when used as a monotherapy compared with the best supportive care in the second-line treatment of advanced GC [63]. The phase III RAINBOW study, which is investigating ramucirumab in combination with paclitaxel in the second-line setting (http://clinicalTrials.gov/ show/NCT01170663), has completed recruitment and results are awaited.

Another key regulator of cell proliferation, growth, survival, metabolism, and angiogenesis is the PI3K/Akt/ mTOR pathway, which is dysregulated in 50-60\% of gastric cancers. Everolimus, an oral mTOR inhibitor, failed to improve OS in patients treated with 1 or 2 lines of systemic chemotherapy when given as monotherapy, but did improve PFS [64]. A phase III trial of the German Arbeitsgemeinschaft Internistische Onkologie (AIO-STO 0111) is currently evaluating paclitaxel with and without everolimus in patients with GC after initial treatment with a fluoropyrimidine-containing regimen (http://clinicalTrials. gov/show/NCT01248403).

The published randomized controlled trials involving molecularly targeted drugs in advanced gastric cancer are delineated in Table 4.

Another target in GC is the receptor tyrosine kinase MET. Met receptor overexpression is associated with poor prognosis for gastric cancer patients. Preliminary results of a randomized phase II trial with rilotumumab, a fully human monoclonal antibody against the Met receptor ligand hepatocyte growth factor, showed improved OS and PFS in patients with high MET expression when combined with ECX [65]. Phase III studies evaluating the clinical benefit of MET inhibitors are under underway.

\section{Post-progression treatment}

Post-progression chemotherapy is effective in advanced gastric cancer. Three randomized controlled trials showed superior survival of patients on either irinotecan or docetaxel monotherapy compared with those receiving best supportive care $[16,17,66]$. In the smallest study, which was performed in Germany, it was reported that post-progression chemotherapy not only prolonged survival but also led to better symptom control. Reports of appropriate quality-of-life measurements are, however, missing in 
Table 4 Reported phase III trials investigating biologically targeted agents in advanced gastric cancer

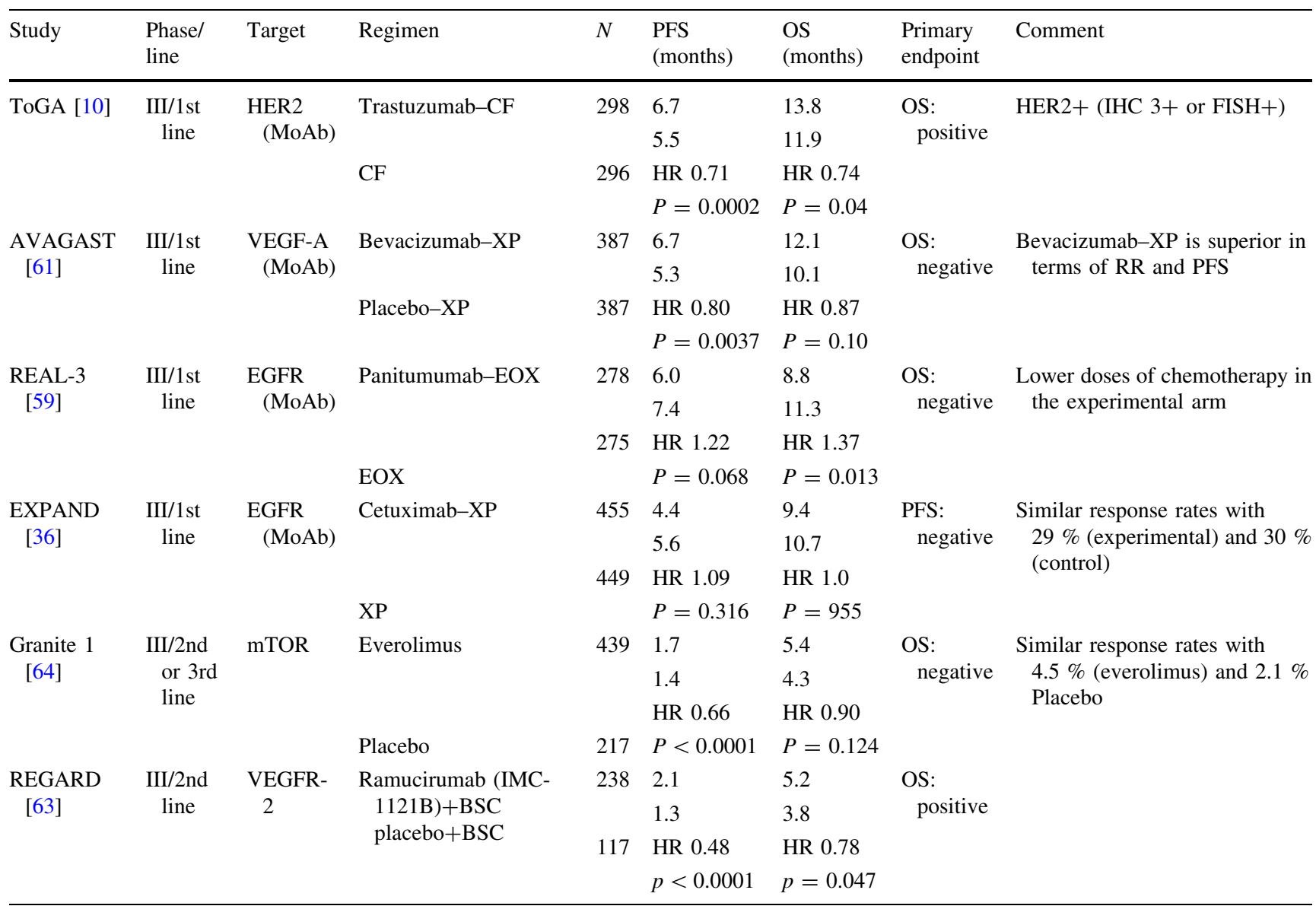

$B S C$ best supportive care, $C F$ cisplatin/fluorouracil, $X P$ capecitabine/cisplatin, $E O X$ epirubicin/oxaliplatin/capecitabine, $M o A B$ monoclonal antibody, $P F S$ progression-free survival, $O S$ overall survival

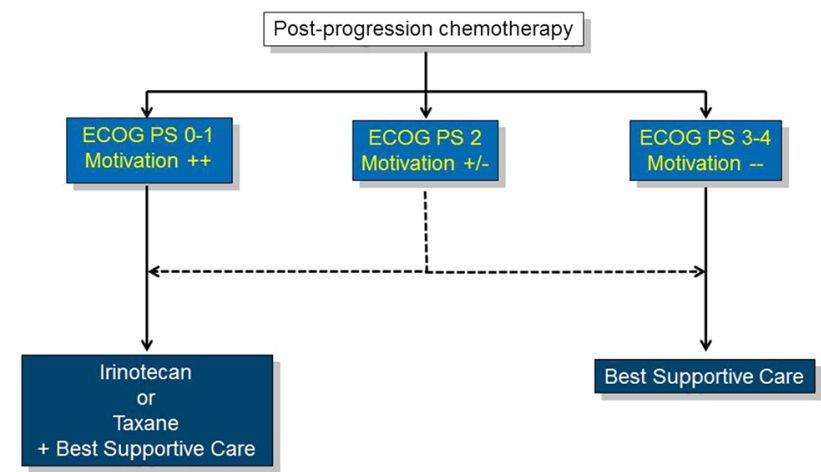

Fig. 3 Indication for post-progression chemotherapy. ECOG PS Eastern Cooperative Group performance status

these studies. A study from the West Japan Oncology Group has recently shown that weekly paclitaxel may also be used in second-line advanced GC [67]. Assessment of palliative treatment goals such as general health status, clinical benefit, and quality of life must be included in further studies [68].
Second-line chemotherapy is now considered a standard therapy option for patients who progress during or after first-line chemotherapy, who are defined as having Eastern Cooperative Group (ECOG) performance status 0-1(2), and who have motivation to be further treated with chemotherapy. Irinotecan administered every 2 or 3 weeks, docetaxel given once every 3 weeks, or weekly paclitaxel are potential options (Fig. 3). Ramucirumab has also been shown to prolong the survival time with a very reasonable side-effect profile [59], but this anti-angiogenic antibody is not yet on the market.

Future outlook

The more we learn about the biological heterogeneity of gastric cancer, the more we can see that there is no single medical treatment that is the best option for all types of gastric cancers. Even with classical cytotoxic treatment, different sensitivities to specific agents may exist in intrinsic GC subtypes [9]. Histologically, distal gastric cancer is classified into intestinal, diffuse, and signet-ring types, and 
adenocarcinoma of the esophagogastric junction is now classified into three unique subsets of upper gastrointestinal adenocarcinoma. With regards to targeted therapy, the development of trastuzumab in HER2-overexpressing gastric cancer raises hope that further progress may be achieved. New targeted agents are under investigation, and some look promising; with better genetic or epigenetic characterization of GC, new and improved treatment options may become available in the future. The identification of biomarkers is essential in order to target the appropriate populations in the trials. Therefore, the collaboration between basic science and clinical research and the performance of well-designed bench-to-bedside studies will be key to achieving further progress in the treatment of advanced gastric cancer.

\section{References}

1. Jemal A, Bray F, Center MM, Ferlay J, Ward E, Forman D, et al. Global cancer statistics. CA Cancer J Clin. 2011;61(2):69-90.

2. Lozano R, Naghavi M, Foreman K, et al. Global and regional mortality from 235 causes of death for 20 age groups in 1990 and 2010: a systematic analysis for the Global Burden of Disease Study 2010. Lancet. 2012;380:2095-128.

3. Kamangar F, Dores GM, Anderson WF. Patterns of cancer incidence, mortality, and prevalence across five continents: defining priorities to reduce cancer disparities in different geographic regions of the world. J Clin Oncol. 2006;24(14):2137-50.

4. Wesolowski R, Lee C, Kim R. Is there a role for second-line chemotherapy in advanced gastric cancer? Lancet Oncol. 2009; 10(9):903-12.

5. Siegel R, DeSantis C, Virgo K, Stein K, Mariotto A, Smith T, et al. Cancer treatment and survivorship statistics, 2012. CA Cancer J Clin. 2012;62(4):220-41.

6. Sakuramoto S, Sasako M, Yamaguchi T, Kinoshita T, Fujii M, Nashimoto A, et al. Adjuvant chemotherapy for gastric cancer with S-1, an oral fluoropyrimidine. N Engl J Med. 2007;357(18): 1810-20.

7. Bang YJ, Kim YW, Yang HK, Chung HC, Park YK, Lee KH, et al. Adjuvant capecitabine and oxaliplatin for gastric cancer after D2 gastrectomy (CLASSIC): a phase 3 open-label, randomised controlled trial. Lancet. 2012;379(9813):315-21.

8. Henson DE, Dittus C, Younes M, Nguyen H, Albores-Saavedra J, et al. Differential trends in the intestinal and diffuse types of gastric carcinoma in the United States, 1973-2000: increase in the signet ring cell type. Arch Pathol Lab Med. 2004;128(7): $765-70$.

9. Tan IB, Ivanova T, Lim KH, Ong CW, Deng N, Lee J, Tan SH, Wu J, Lee $\mathrm{MH}$, Ooi $\mathrm{CH}$, Rha SY, et al. Intrinsic subtypes of gastric cancer, based on gene expression pattern, predict survival and respond differently to chemotherapy. Gastroenterology. 2011;141(2):476-85 485 e471-411.

10. Bang YJ, Van Cutsem E, Feyereislova A, Chung HC, Shen L, Sawaki A, et al. Trastuzumab in combination with chemotherapy versus chemotherapy alone for treatment of HER2-positive advanced gastric or gastro-oesophageal junction cancer (ToGA): a phase 3, open-label, randomised controlled trial. Lancet. 2010; 376(9742):687-97.

11. Deng N, Goh LK, Wang H, Das K, Tao J, Tan IB, et al. A comprehensive survey of genomic alterations in gastric cancer reveals systematic patterns of molecular exclusivity and co- occurrence among distinct therapeutic targets. Gut. 2012;61(5): 673-84.

12. Wagner AD, Unverzagt S, Grothe W, Kleber G, Grothey A, Haerting J, et al. Chemotherapy for advanced gastric cancer. Cochrane Database Syst Rev. 2010; (3):CD004064.

13. Pyrhonen S, Kuitunen T, Nyandoto P, Kouri M, et al. Randomised comparison of fluorouracil, epidoxorubicin and methotrexate (FEMTX) plus supportive care with supportive care alone in patients with non-resectable gastric cancer. Br J Cancer. 1995; 71(3):587-91.

14. Murad AM, Santiago FF, Petroianu A, Rocha PR, Rodrigues MA, Rausch M, et al. Modified therapy with 5-fluorouracil, doxorubicin, and methotrexate in advanced gastric cancer. Cancer. 1993;72(1):37-41.

15. Glimelius B, Ekstrom K, Hoffman K, Graf W, Sjoden PO, Haglund U, et al. Randomized comparison between chemotherapy plus best supportive care with best supportive care in advanced gastric cancer. Ann Oncol. 1997;8(2):163-8.

16. Thuss-Patience PC, Kretzschmar A, Bichev D, Deist T, Hinke A, Breithaupt K, et al. Survival advantage for irinotecan versus best supportive care as second-line chemotherapy in gastric cancer-a randomised phase III study of the Arbeitsgemeinschaft Internistische Onkologie (AIO). Eur J Cancer. 2011;47(15):2306-14.

17. Kang JH, Lee SI, Lim do H, Park KW, Oh SY, Kwon HC, et al. Salvage chemotherapy for pretreated gastric cancer: a randomized phase III trial comparing chemotherapy plus best supportive care with best supportive care alone. J Clin Oncol. 2012;30(13): 1513-8.

18. Lutz MP, Wilke H, Wagener DJ, Vanhoefer U, Jeziorski K, Hegewisch-Becker S, et al. Weekly infusional high-dose fluorouracil (HD-FU), HD-FU plus folinic acid (HD-FU/FA), or HDFU/FA plus biweekly cisplatin in advanced gastric cancer: randomized phase II trial 40953 of the European Organisation for Research and Treatment of Cancer Gastrointestinal Group and the Arbeitsgemeinschaft Internistische Onkologie. J Clin Oncol. 2007;25(18):2580-5.

19. Koizumi W, Narahara H, Hara T, Takagane A, Akiya T, Takagi $\mathrm{M}$, et al. S-1 plus cisplatin versus S-1 alone for first-line treatment of advanced gastric cancer (SPIRITS trial): a phase III trial. Lancet Oncol. 2008;9(3):215-21.

20. Trumper M, Ross PJ, Cunningham D, Norman AR, Hawkins R, Seymour M, et al. Efficacy and tolerability of chemotherapy in elderly patients with advanced oesophago-gastric cancer: a pooled analysis of three clinical trials. Eur J Cancer. 2006;42(7): 827-34.

21. Preusser P, Wilke H, Achterrath W, Lenaz L, Stahl M, Casper J, et al. Phase II study of carboplatin in untreated inoperable advanced stomach cancer. Eur J Cancer. 1990;26(10):1108-9.

22. Louvet C, Andre T, Tigaud JM, Gamelin E, Douillard JY, Brunet $\mathrm{R}$, et al. Phase II study of oxaliplatin, fluorouracil, and folinic acid in locally advanced or metastatic gastric cancer patients. J Clin Oncol. 2002;20(23):4543-8.

23. Al-Batran SE, Atmaca A, Hegewisch-Becker S, Jaeger D, Hahnfeld S, Rummel MJ, et al. Phase II trial of biweekly infusional fluorouracil, folinic acid, and oxaliplatin in patients with advanced gastric cancer. J Clin Oncol. 2004;22(4):658-63.

24. Lordick F, Lorenzen S, Stollfuss J, Vehling-Kaiser U, Kullmann F, Hentrich M, et al. Phase II study of weekly oxaliplatin plus infusional fluorouracil and folinic acid (FUFOX regimen) as firstline treatment in metastatic gastric cancer. Br J Cancer. 2005;93 (2):190-4.

25. Cunningham D, Starling N, Rao S, Iveson T, Nicolson M, Coxon F, et al. Capecitabine and oxaliplatin for advanced esophagogastric cancer. N Engl J Med. 2008;358(1):36-46.

26. Al-Batran SE, Hartmann JT, Probst S, Schmalenberg H, Hollerbach S, Hofheinz R, et al. Phase III trial in metastatic 
gastroesophageal adenocarcinoma with fluorouracil, leucovorin plus either oxaliplatin or cisplatin: a study of the Arbeitsgemeinschaft Internistische Onkologie. J Clin Oncol. 2008;26(9): 1435-42.

27. Koizumi W, Takiuchi H, Yamada Y, Boku N, Fuse N, Muro K, et al. Phase II study of oxaliplatin plus S-1 as first-line treatment for advanced gastric cancer (G-SOX study). Ann Oncol. 2010;21(5):1001-5.

28. Kang YK, Kang WK, Shin DB, Chen J, Xiong J, Wang J, et al. Capecitabine/cisplatin versus 5-fluorouracil/cisplatin as first-line therapy in patients with advanced gastric cancer: a randomised phase III noninferiority trial. Ann Oncol. 2009;20(4):666-73.

29. Okines AF, Norman AR, McCloud P, Kang YK, Cunningham D, et al. Meta-analysis of the REAL-2 and ML17032 trials: evaluating capecitabine-based combination chemotherapy and infused 5-fluorouracil-based combination chemotherapy for the treatment of advanced oesophago-gastric cancer. Ann Oncol. 2009;20(9): 1529-34.

30. Boku N, Yamamoto S, Fukuda H, Shirao K, Doi T, Sawaki A, et al. Fluorouracil versus combination of irinotecan plus cisplatin versus S-1 in metastatic gastric cancer: a randomised phase 3 study. Lancet Oncol. 2009;10(11):1063-9.

31. Ajani JA, Faust J, Ikeda K, Yao JC, Anbe H, Carr KL, et al. Phase I pharmacokinetic study of S-1 plus cisplatin in patients with advanced gastric carcinoma. J Clin Oncol. 2005;23(28):6957-65.

32. Ajani JA, Rodriguez W, Bodoky G, Moiseyenko V, Lichinitser M, Gorbunova V, et al. Multicenter phase III comparison of cisplatin/S-1 with cisplatin/infusional fluorouracil in advanced gastric or gastroesophageal adenocarcinoma study: the FLAGS trial. J Clin Oncol. 2010;28(9):1547-53.

33. Yamaguchi K, Sawaki A, Doi T, Satoh T, Yamada Y, Omuro Y, et al. Efficacy and safety of capecitabine plus cisplatin in Japanese patients with advanced or metastatic gastric cancer: subset analyses of the AVAGAST study and the ToGA study. Gastric Cancer. 2013;16(2):175-82.

34. Ma BB, Hui EP, Mok TS. Population-based differences in treatment outcome following anticancer drug therapies. Lancet Oncol. 2010;11(1):75-84.

35. Ohtsu A, Shah MA, Van Cutsem E, Rha SY, Sawaki A, Park SR, et al. Bevacizumab in combination with chemotherapy as firstline therapy in advanced gastric cancer: a randomized, doubleblind, placebo-controlled phase III study. J Clin Oncol. 2011; 29(30):3968-76.

36. Lordick F, Kang YK, Chung HC, Salman P, Oh SC, Bodoky G, et al. Capecitabine and cisplatin with or without cetuximab for patients with previously untreated advanced gastric cancer (EXPAND): a randomised, open-label phase 3 trial. Lancet Oncol. 2013;14(6):490-9

37. Tebbutt NC, Norman A, Cunningham D, Iveson T, Seymour M, Hickish $\mathrm{T}$, et al. A multicentre, randomised phase III trial comparing protracted venous infusion (PVI) 5-fluorouracil (5-FU) with PVI 5-FU plus mitomycin $\mathrm{C}$ in patients with inoperable oesophago-gastric cancer. Ann Oncol. 2002;13(10):1568-75.

38. Cullinan SA, Moertel CG, Wieand HS, O'Connell MJ, Poon MA, Krook JE, et al. Controlled evaluation of three drug combination regimens versus fluorouracil alone for the therapy of advanced gastric cancer. North Central Cancer Treatment Group. J Clin Oncol. 1994;12(2):412-6.

39. Ross P, Nicolson M, Cunningham D, Valle J, Seymour M, Harper $\mathrm{P}$, et al. Prospective randomized trial comparing mitomycin, cisplatin, and protracted venous-infusion fluorouracil (PVI 5-FU) With epirubicin, cisplatin, and PVI 5-FU in advanced esophagogastric cancer. J Clin Oncol. 2002;20(8):1996-2004.

40. Yun J, Lee J, Park SH, Park JO, Park YS, Lim HY, et al. A randomised phase II study of combination chemotherapy with epirubicin, cisplatin and capecitabine (ECX) or cisplatin and capecitabine $(\mathrm{CX})$ in advanced gastric cancer. Eur $\mathrm{J}$ Cancer. 2010;46(5):885-91.

41. Van Cutsem E, Moiseyenko VM, Tjulandin S, Majlis A, Constenla M, Boni C, et al. Phase III study of docetaxel and cisplatin plus fluorouracil compared with cisplatin and fluorouracil as firstline therapy for advanced gastric cancer: a report of the V325 Study Group. J Clin Oncol. 2006;24(31):4991-7.

42. Lorenzen S, Hentrich M, Haberl C, Heinemann V, Schuster T, Seroneit T, et al. Split-dose docetaxel, cisplatin and leucovorin/ fluorouracil as first-line therapy in advanced gastric cancer and adenocarcinoma of the gastroesophageal junction: results of a phase II trial. Ann Oncol. 2007;18(10):1673-9.

43. Al-Batran SE, Hartmann JT, Hofheinz R, Homann N, Rethwisch $\mathrm{V}$, Probst S, et al. Biweekly fluorouracil, leucovorin, oxaliplatin, and docetaxel (FLOT) for patients with metastatic adenocarcinoma of the stomach or esophagogastric junction: a phase II trial of the Arbeitsgemeinschaft Internistische Onkologie. Ann Oncol. 2008;19(11):1882-7.

44. Tebbutt NC, Cummins MM, Sourjina T, Strickland A, Van Hazel G, Ganju V, et al. Randomised, non-comparative phase II study of weekly docetaxel with cisplatin and 5-fluorouracil or with capecitabine in oesophagogastric cancer: the AGITG ATTAX trial. Br J Cancer. 2010;102(3):475-81.

45. Al-Batran SE, Pauligk C, Homann N, Hartmann JT, Moehler M, Probst S, Rethwisch V, et al. The feasibility of triple-drug chemotherapy combination in older adult patients with oesophagogastric cancer: a randomised trial of the Arbeitsgemeinschaft Internistische Onkologie (FLOT65+). Eur J Cancer. 2013;49(4): $835-42$

46. Yoshida K, Ninomiya M, Takakura N, Hirabayashi N, Takiyama $\mathrm{W}$, Sato Y, et al. Phase II study of docetaxel and S-1 combination therapy for advanced or recurrent gastric cancer. Clin Cancer Res. 2006;12(11 Pt 1):3402-7.

47. Yoshida K, Fujii M, Koizumi W, Kim H, Kim Y, Takeuchi M. S-1 plus Docetaxel versus S-1 for Advanced Gastric Cancer (START Trial) Update 2012 (JACCRO and KCSG study Group). Ann Oncol 2012;23(Suppl 9):LBA19_PR (abstract 1317).

48. Koizumi W, Nakayama N, Tanabe S, Sasaki T, Higuchi K, Nishimura $\mathrm{K}$, et al. A multicenter phase II study of combined chemotherapy with docetaxel, cisplatin, and S-1 in patients with unresectable or recurrent gastric cancer (KDOG 0601). Cancer Chemother Pharmacol. 2012;69(2):407-13.

49. Pozzo C, Barone C, Szanto J, Padi E, Peschel C, Bukki J, et al. Irinotecan in combination with 5-fluorouracil and folinic acid or with cisplatin in patients with advanced gastric or esophagealgastric junction adenocarcinoma: results of a randomized phase II study. Ann Oncol. 2004;15(12):1773-81.

50. Moehler M, Eimermacher A, Siebler J, Hohler T, Wein A, Menges M, et al. Randomised phase II evaluation of irinotecan plus high-dose 5-fluorouracil and leucovorin (ILF) vs 5-fluorouracil, leucovorin, and etoposide (ELF) in untreated metastatic gastric cancer. Br J Cancer. 2005;92(12):2122-8.

51. Dank M, Zaluski J, Barone C, Valvere V, Yalcin S, Peschel C, et al. Randomized phase III study comparing irinotecan combined with 5-fluorouracil and folinic acid to cisplatin combined with 5 -fluorouracil in chemotherapy naive patients with advanced adenocarcinoma of the stomach or esophagogastric junction. Ann Oncol. 2008;19(8):1450-7.

52. Moehler M, Kanzler S, Geissler M, Raedle J, Ebert MP, Daum S, et al. A randomized multicenter phase II study comparing capecitabine with irinotecan or cisplatin in metastatic adenocarcinoma of the stomach or esophagogastric junction. Ann Oncol. 2010; 21(1):71-7.

53. Ajani JA, Moiseyenko VM, Tjulandin S, Majlis A, Constenla M, Boni $\mathrm{C}$, et al. Quality of life with docetaxel plus cisplatin and fluorouracil compared with cisplatin and fluorouracil from a 
phase III trial for advanced gastric or gastroesophageal adenocarcinoma: the V-325 Study Group. J Clin Oncol. 2007;25(22): 3210-6.

54. Roth AD, Fazio N, Stupp R, Falk S, Bernhard J, Saletti P, et al. Docetaxel, cisplatin, and fluorouracil; docetaxel and cisplatin; and epirubicin, cisplatin, and fluorouracil as systemic treatment for advanced gastric carcinoma: a randomized phase II trial of the Swiss Group for Clinical Cancer Research. J Clin Oncol. 2007;25(22):3217-23.

55. Lordick F. HER2 in gastric cancer: a biomarker with clinical impact, but not without translational challenges. Clin Transl Oncol. 2011;13(9):597-8.

56. Warneke VS, Behrens HM, Böger C, Becker T, Lordick F, Ebert MP, Röcken C. Her2/neu testing in gastric cancer: evaluating the risk of sampling errors. Ann Oncol. 2013;24(3):725-33.

57. Iqbal S, Goldman B, Fenoglio-Preiser CM, Lenz HJ, Zhang W, Danenberg KD, et al. Southwest Oncology Group study S0413: a phase II trial of lapatinib (GW572016) as first-line therapy in patients with advanced or metastatic gastric cancer. Ann Oncol. 2011;22(12):2610-5.

58. Hecht JR, Bang YJ, Qin S. Lapatinib in combination with capecitabine plus oxaliplatin (CapeOx) in HER2-positive advanced or metastatic gastric, esophageal, or gastroesophageal adenocarcinoma (AC): The TRIO-013/LOGiC Trial. J Clin Oncol 31, 2013 (suppl; abstr LBA4001). 2013.

59. Waddell T, Chau I, Cunningham D, Gonzalez D, Okines AF, Okines C, et al. Epirubicin, oxaliplatin, and capecitabine with or without panitumumab for patients with previously untreated advanced oesophagogastric cancer (REAL3): a randomised, open-label phase 3 trial. Lancet Oncol. 2013;14(6):481-9.

60. Luber B, Deplazes J, Keller G, Walch A, Rauser S, Eichmann M, et al. Biomarker analysis of cetuximab plus oxaliplatin/leucovorin/5-fluorouracil in first-line metastatic gastric and oesophagogastric junction cancer: results from a phase II trial of the $\mathrm{Ar}$ beitsgemeinschaft Internistische Onkologie (AIO). BMC Cancer. 2011;11:509.

61. Van Cutsem E, de Haas S, Kang YK, Ohtsu A, Tebbutt NC, Ming $\mathrm{Xu} \mathrm{J}$, et al. Bevacizumab in combination with chemotherapy as first-line therapy in advanced gastric cancer: a biomarker evaluation from the AVAGAST randomized phase III trial. J Clin Oncol. 2012;30(17):2119-27.

62. Spratlin JL, Cohen RB, Eadens M, Gore L, Camidge DR, Diab S, et al. Phase I pharmacologic and biologic study of ramucirumab (IMC-1121B), a fully human immunoglobulin G1 monoclonal antibody targeting the vascular endothelial growth factor receptor-2. J Clin Oncol. 2010;28(5):780-7.

63. Fuchs CS, Tomasek J, Cho JY, Dumitro F, Passalacqua R, Goswami C. REGARD: A phase III, randomized, double-blinded trial of ramucirumab and best supportive care (BSC) versus placebo and BSC in the treatment of metastatic gastric or gastroesophageal junction (GEJ) adenocarcinoma following disease progression on first-line platinum- and/or fluoropyrimidine-containing combination chemotherapy. J Clin Oncol 2013; 30. suppl 34; abstr LBA5.

64. van Cutsem E, Yeh KH, Bang YJ, Shen L, Ajani JA, Bai YX, et al. Phase III trial of everolimus (EVE) in previously treated patients with advanced gastric cancer (AGC): GRANITE-1. J Clin Oncol 30, (suppl 4; abstr LBA3). 2012.

65. Oliner KS, Tang R, Anderson A. Evaluation of MET pathway biomarkers in a phase II study of rilotumumab (R, AMG 102) or placebo $(\mathrm{P})$ in combination with epirubicine, cisplatin and capecitabine (ECX) in patients with locally advanced or metastatic gastric (G)or esophagogastric junction (EGJ) cancer. J Clin Oncol. 2012; 30. abstr 4005.

66. Ford H, Marshall A, Wadsley J. Cougar-02: a randomized phase III study of docetaxel versus active symptom control in advanced esophagogastric adenocarcinoma. J Clin Oncol. 2013; 30. suppl 34 (abstr LBA4).

67. Ueda S, Hironaka S, Yasui H, Nishina T, Tsuda M, Tsumura T. Randomized phase III study of irinotecan (CPT-11) versus weekly paclitaxel (wPTX) for advanced gastric cancer (AGC) refractory to combination chemotherapy (CT) of fluoropyrimidine plus platinum (FP): WCOG4007 trial. J Clin Oncol 2012; 30 (suppl; abstr 4002).

68. Lordick F. Gastrointestinal cancer: salvage chemotherapy in gastric cancer-more than a straw? Nat Rev Clin Oncol. 2012; 9(6):312-3.

69. Shah M, Shibata S, Stoller RG, Kemeny M, Ritch PS, Krishnamurthi SS. Random assignment multicenter phase II study of modified docetaxel, cisplatin, fluorouracil (mDCF) versus DCF with growth factor support (GCSF) in metastatic gastroesophageal adenocarcinoma (GE) [abstract]. J Clin Oncol. 2010:s28: 4014.

70. van Cutsem E, Boni C, Tabernero J, Massuti B, Richards DA, Prenen H, et al. Randomized phase II study (GATE study) of docetaxel plus oxaliplatin with or without fluorouracil or capecitabine in metastatic or locally recurrent gastric cancer. J Clin Oncol 29: (suppl; abstr 4018). 2011. 